\title{
Exposure to the environmental pollutant bisphenol A diglycidyl ether (BADGE) causes cell over-proliferation in Drosophila
}

\author{
Michael J. Williams ${ }^{1} \cdot$ Hao Cao ${ }^{1} \cdot$ Therese Lindkvist $^{1} \cdot$ Tobias J. Mothes $^{1} \cdot$ Helgi B. Schiöth ${ }^{1,2}$ \\ Received: 5 December 2019/Accepted: 14 April 2020/Published online: 28 April 2020 \\ (C) The Author(s) 2020
}

\begin{abstract}
Bisphenol A diglycidyl ether (BADGE), a derivative of bisphenol A (BPA), is widely used in the manufacture of epoxy resins as well as a coating on food containers. Recent studies have demonstrated the adverse effects of BADGE on reproduction and development in rodents and amphibians, but how BADGE affects biological activity is not understood. To gain a better understanding of the biological effects of BADGE exposure during development, we used the model organism Drosophila melanogaster and performed whole transcriptome sequencing. Interestingly, when Drosophila are raised on food containing BADGE, genes having significantly increased transcript numbers are enriched for those involved in regulating cell proliferation, including DNA replication and cell cycle control. Furthermore, raising larvae on BADGE-containing food induces hemocyte (blood cell) over-proliferation. This effect can be stimulated with even lower concentrations of BADGE if the hemocytes are already primed for cell proliferation by the expression of dominant active Ras GTPase. We conclude that chronic exposure to the xenobiotic BADGE throughout development can induce cell proliferation.
\end{abstract}

Keywords Whole transcriptome sequencing $\cdot$ Environmental pollutant $\cdot$ Cell cycle $\cdot$ Hematopoiesis $\cdot$ Hemocyte

\section{Introduction}

By 2015, the global production of plastics reached 380 million metric tons annually (Geyer et al. 2017; Jambeck et al. 2015) and the tonnage continues to increase. Bisphenols, which have been in commercial use as plasticizers since the 1950s, are reported as estrogenic mimics and may interfere with hormonal activity, especially during gestational and early developmental stages. Furthermore, several bisphenols have been shown to have neurotoxic properties or are proposed to be carcinogenic (Jadhav et al. 2017; Pang et al. 2019).

Responsible editor: Mohamed M. Abdel-Daim

Electronic supplementary material The online version of this article (https://doi.org/10.1007/s11356-020-08899-7) contains supplementary material, which is available to authorized users.

Michael J. Williams

michael.williams@neuro.uu.se

1 Department of Neuroscience, Functional Pharmacology, Uppsala University, Husargatan 3, Box 593, 75124 Uppsala, Sweden

2 Institute for Translational Medicine and Biotechnology, Sechenov First Moscow State Medical University, Moscow, Russia
Bisphenol A diglycidyl ether (BADGE), an epoxy resin produced through the reaction of bisphenol A (BPA) and epichlorohydrin, is found in canned food and beverages, as well as in paints and adhesives, where it is used as an internal varnish to prevent degradation (Poole et al. 2004). During the manufacturing process there is some residual BADGE not covalently bound to the epoxy resin. This means the pollutant can easily leach from the container to contaminate food and beverages. Moreover, upon contact with aqueous or acidic matrices, BADGE can be hydrolyzed to form derivatives, such as BADGE $\cdot \mathrm{H}_{2} \mathrm{O}$ and BADGE $2 \mathrm{H}_{2} \mathrm{O}$ (Suarez et al. 2000). Additionally, the presence of hydrochloric acid in organosol lacquers promotes the formation of chlorohydroxy derivatives, including $\mathrm{BADGE} \cdot 2 \mathrm{HCl}, \mathrm{BADGE} \cdot \mathrm{HCl}$, and BADGE $\cdot \mathrm{H}_{2} \mathrm{O} \cdot \mathrm{HCl}$ (Hammarling et al. 2010). Many of these derivatives have been shown to induce both cytotoxic and genotoxic effects (Suarez et al. 2000).

Even though BADGE is manufactured from BPA, studies indicate that when BADGE is metabolized, it is not a significant source of BPA (Climie et al. 1981). BADGE and its hydrolytic derivatives have been found in human urine, plasma, and adipose fat (Wang et al. 2012; Wang et al. 2015). Due to the fact that BADGE is easily hydrolyzed in water or chlorinated in chlorine-containing coating solutions (Yonekubo 
et al. 2008), its concentration is difficult to detect in epoxycoated commodities, and therefore, its disrupting effects can be underestimated. Similar to BPA, the estrogenic activity and reproductive/developmental toxicity of BADGE have been demonstrated (Hyoung et al. 2007; Nakazawa et al. 2002).

The toxicity of the BPA has been extensively studied, yet current information on the physiological and molecular effects of BADGE is scarce (Poole et al. 2004). Furthermore, the reported effects of BADGE on cell proliferation have been contradictory. For instance, BADGE was reported to decrease cell proliferation and induce morphological changes, as well as cell detachment, in human colorectal adenocarcinoma Caco-2 cells (Ramilo et al. 2006). On the other hand, BADGE, and its chlorohydroxy derivatives, triggered proliferation in human breast cancer T47D cells (Nakazawa et al. 2002). There is also evidence that through its inhibition of the nuclear receptor peroxisome proliferator-activated receptorgamma (PPAR $\gamma$ ) BADGE may drive cells towards proliferation at the exclusion of cellular differentiation (Zhu et al. 2013).

Most studies on the effects of BADGE have been performed in cell lines. In our study, we employed the model organism Drosophila melanogaster as the experimental subject to gain a better understanding of the xenobiotic effects of BADGE exposure in vivo. To do this, we performed whole transcriptome sequencing on RNA from flies exposed to BADGE throughout development. This provided a number of lead candidate genes involved in the effect of BADGE and several of these leads were verified in expression analysis. Moreover, we performed physiological studies providing further functional information on the effect of BADGE.

\section{Materials and methods}

Fly strain and maintenance The $w^{1118} ; P\{w[+m C]=H m l-$ GAL4.Delta $\} 2, P\{w[+m C]=U A S-2 x E G F P\} A H 2(H m l-$ GFP) and $w^{*} ; P\{w[+m C]=U A S-R a s 85 D . V 12\} 2$, (UAS$\operatorname{Ras}^{V 12}$ ) strains were provided by the Bloomington Drosophila Stock Center (Bloomington, IN, USA). The CantonS and OregonR-C flies, also obtained from the Bloomington Drosophila Stock Center, were crossed to create the CSORC laboratory wild-type strain. All fly stocks were raised on Jazz-mix Drosophila food (Fisher Scientific) and supplemented with yeast extract (Genesee Scientific, San Diego, CA, USA). The cultures were maintained at $25{ }^{\circ} \mathrm{C}$ and $60 \%$ humidity on a 12:12 light/dark cycle. The adult male flies for experiments, unless otherwise stated, were collected after eclosion and aged for 5-7 days. Since the Hml-GFP flies express both green fluorescent protein and GAL4 in hemocytes, the Hml-GFP strain was crossed to UAS-Ras ${ }^{V 12}$ to overexpress Ras. For examining the hemocyte by bleeding assay, no further staining was applied.
Bisphenol A diglycidyl ether feeding The BADGE (SigmaAldrich, Stockholm, Sweden) solutions were dissolved in 99\% ethanol, and either $1 \mathrm{ml}$ of BADGE solutions or ethanol was diluted in $50 \mathrm{ml}$ Jazz-mix Drosophila food. The final BADGE concentrations in fly food were $20 \mu \mathrm{M}$ (or $135 \mu \mathrm{M}$ for live imaging), $60 \mu \mathrm{M}$, and $200 \mu \mathrm{M}$, respectively. After BADGE-enriched food preparation, the female and male flies (3:1 ratio) were placed in pre-prepared bottles and allowed to lay eggs on the food. The larvae were continuously raised on BADGE-enriched or control food until they eclosed. When the progeny eclosed, male flies were collected and transferred to a vial with $6 \mathrm{ml}$ of BADGE-enriched food for further uses.

mRNA preparation and SOLiD sequencing The mRNA was isolated using Dynabeads mRNA purification kit (Thermo Fisher, Sweden) (Williams et al. 2016). The flies used for sequencing were fed on either control or $20 \mu \mathrm{M}$ BADGEenriched food. Three biological replicates, which contained 10 whole flies in each replicate, were prepared for sequencing. As we described previously (Williams et al. 2016), the RNAseq data were analyzed using TopHat and Cufflinks. To test the differential expression of genes, the pre-analyzed data were further evaluated using the Cuffcompare and Cuffdiff tools. The calculated $p$ value and $q$ value (the FDR-adjusted $p$ value of the test statistic) from Cuffdiff were used. Only genes with $q<0.05$ and $\log 2> \pm 1$-fold change were considered as significantly affected.

\section{RNA purification, CDNA synthesis, and qRT-PCR}

\section{RNA}

The phenol-chloroform method was used for RNA extraction from tissue samples (Williams et al. 2013). Ten whole male flies, 5-7 days old, were homogenized with $800 \mu \mathrm{l}$ TRIzol (Thermo Fisher, Sweden), $200 \mu \mathrm{l}$ chloroform (Sigma-Aldrich, Sweden) was added, and samples were centrifuged at $12000 \mathrm{rpm}$ for $15 \mathrm{~min}$ at $4{ }^{\circ} \mathrm{C}$. The aqueous layer, which contained RNA, was separated and $500 \mu$ isopropanol (Solvaco AB, Sweden) was added. The RNA was precipitated by storing the samples at $-32^{\circ} \mathrm{C}$ for $2 \mathrm{~h}$. Samples were centrifuged at $12,000 \mathrm{rpm}$ for $10 \mathrm{~min}$ at $4{ }^{\circ} \mathrm{C}$, to collect the RNA pellets, which were then washed with $75 \%$ ethanol (Solvaco $\mathrm{AB}$, Sweden) to remove the organic impurities. Samples were allowed to air dry to remove any traces of ethanol. Dried RNA pellets were dissolved in $21.4 \mu \mathrm{l}$ of RNAse-free water (Qiagen $\mathrm{GmBH}$, Germany) and $2.6 \mu \mathrm{l}$ of DNAse incubation buffer (Roche GmBH, Germany). The samples were incubated at $75{ }^{\circ} \mathrm{C}$ for 15 min to ensure complete dissolution of RNApellets. Two microliters of DNAse I (10 U/ $\mu \mathrm{L}$, Roche $\mathrm{GmBH}$, Germany) was added to each sample and incubated at $37^{\circ} \mathrm{C}$ for $3 \mathrm{~h}$ to remove DNA contamination. DNAse was 
deactivated by incubating the samples at $75{ }^{\circ} \mathrm{C}$ for $15 \mathrm{~min}$. The removal of DNA was confirmed by PCR using Taq polymerase ( $5 \mathrm{U} / \mu \mathrm{l}$, Biotools B \& M Labs, Spain), followed by agarose gel electrophoresis. The RNA concentration was measured using a nanodrop ND 1000 spectrophotometer (Saveen Werner, Sweden).

\section{cDNA synthesis}

cDNA was synthesized from RNA template using dNTP $20 \mathrm{mM}$ (Fermentas Life Science, USA), random hexamer primers, and M-MLV reverse transcriptase $(200 \mathrm{U} / \mu \mathrm{l}$, Invitrogen, USA) by following the manufacturer's instructions. cDNA synthesis was confirmed by PCR followed by agarose gel electrophoresis (Williams et al. 2013).

\section{qRT-PCR}

Relative expression levels of three housekeeping genes (Rp49 and RpL11) and of the genes of interest were determined with quantitative RT-PCR (qPCR) (Williams et al. 2013). Each reaction, with a total volume of $20 \mu \mathrm{l}$, contained $20 \mathrm{mM}$ Tris/ $\mathrm{HCl} \mathrm{pH} 9.0,50 \mathrm{mM} \mathrm{KCl}, 4 \mathrm{mM} \mathrm{MgCl}_{2}, 0.2 \mathrm{mM}$ dNTP, DMSO (1:20), and SYBR Green (1:50000). The template concentration was $5 \mathrm{ng} / \mu \mathrm{l}$ and the concentration of each primer was $2 \mathrm{pmol} / \mu \mathrm{l}$. Primers were designed with Beacon Designer (Premier Biosoft, CA, USA) using the SYBR Green settings. All qPCR experiments were performed in duplicate; for each primer pair, a negative control with water and a positive control with $5 \mathrm{ng} / \mu$ l of genomic DNA were included on each plate. Amplifications were performed with $0.02 \mu \mathrm{g} / \mathrm{ml}$ Taq DNA polymerase (Biotools, Sweden) under the following conditions: initial denaturation at $95^{\circ} \mathrm{C}$ for $3 \mathrm{~min}, 50$ cycles of denaturing at $95{ }^{\circ} \mathrm{C}$ for $15 \mathrm{~s}$, annealing at $52.8-60.1{ }^{\circ} \mathrm{C}$ for $15 \mathrm{~s}$, and extension at $72{ }^{\circ} \mathrm{C}$ for $30 \mathrm{~s}$. Analysis of qPCR data was performed using MyIQ 1.0 software (Bio-Rad) as previously reported. Primer efficiencies were calculated using LinRegPCR (Ramakers et al. 2003) and samples were corrected for differences in primer efficiencies. The GeNorm protocol described by Vandesompele et al. (Vandesompele et al. 2002) was used to calculate normalization factors from the expression levels of the housekeeping genes. Differences in gene expression between groups were analyzed with ANOVA followed by Fisher's PLSD test where appropriate. $p<0.05$ was used as the criterion of statistical significance. The following primers (Thermo Fisher Scientific, Germany) Rp49-F: 5'- CACACCAAATCTTACAAAATGTGTGA-3' Rp49-R: 5'-AATCCGGCCTTGCACATG-3' $C y c B$-F: 5'GTGAACGAGCCCACCTTAAAG-3' $C y c B$-R: 5'-GAAA CTCCCATCACGGGTTTG-3' $C y c E-\mathrm{F}: 5^{\prime}$-AGCC TCCATCAGCTAAGCG-3' $C y c E-\mathrm{R}: 5^{\prime}-\mathrm{GCAA}$ CCGATGACAGATTGCC-3' stg-F : 5'-CAGC AGTTCGAGTAGCATCAA-3' stg-R: 5'-CTCC
CATAGCTGGCAGAATTTT-3' $p n g-\mathrm{F}: 5^{\prime}-\mathrm{GCCA}$ GGGCAAGGTGGATTT- $3^{\prime} p n g-\mathrm{R}: 5^{\prime}$-CCAC ACGGGATCGTAGAGAC-3' $g n u-\mathrm{F}: 5^{\prime}-\mathrm{TAAA}$ TGGGGAGACCAGGCTAC-3' gnu-R: 5'-CGCA CTTTTCAACTTGGATTCCT-3' plu-F: 5'-GGAA TACCGCCCTATTGAAGG-3'plu-R: 5 '-ATCT TCGCCTCAACAGTTCCT-3'.

Larvae bleeding and hemocytes counting Third instar larvae were collected and kept in 1x PBS solution for 5-10 min before bleeding. One drop of 1x PBS solution was placed on a glass slide. To get enough hemocytes, the larva was squeezed by another forceps. After $30 \mathrm{~min}$, the cells were settled and attached to slide. For the CSORC larvae, the hemocytes were stained by DAPI for $20 \mathrm{~min}$. For the $\mathrm{Hml}$ GFP $>U A S$-Ras ${ }^{V 12}$ cross, the flies were raised at $20^{\circ} \mathrm{C}$ in order to inhibit the activity and lethal effect of Ras overexpression. The slides were then mounted and the images were taken by a Zeiss Axioplan 2 microscope. The number of hemocytes was counted using the ImageJ software.

Live imaging Hml-GFP larvae were used for live imaging and maintained on $0,60,135$, and $200 \mu \mathrm{M}$ BADGE food, respectively. Third instar larvae were anesthetized on ice and fixed by a drop transparent nail polish. The slides were immediately imaged using a Zeiss LSM 700 confocal microscope.

Data analysis The data analysis and plotting were performed by Prism Graphpad 5. The data were plotted as mean \pm SEM. Normal distribution was performed using the KolmogorovSmirnov test of normality. All the significant differences were tested following one-way ANOVA with Tukey's post hoc. The significances were represented as $* p<0.05, * * p<0.01$, and $* * * p<0.001$ and also specified in the corresponding figure legend.

\section{Results}

\section{BADGE exposure causes upregulation of cell cycle genes}

To gain a better understanding of how bisphenol A diglycidyl ether (BADGE) influences biological systems in vivo, $\mathrm{F}_{0}$ Drosophila females were allowed to lay eggs on food containing $20 \mu \mathrm{M}$ BADGE (calculated from the human no observed adverse effect level, NOAEL) (Poole et al. 2004). Three replicates of $F_{1}$ progeny were collected, which had been raised on BADGE-containing food from embryos to 5-7-day-old adults. Total RNA was then extracted from male flies for whole transcriptome sequencing. Due to the fact that the reproductive cycle of female flies could potentially influence their behavior, including food intake, we used only adult 


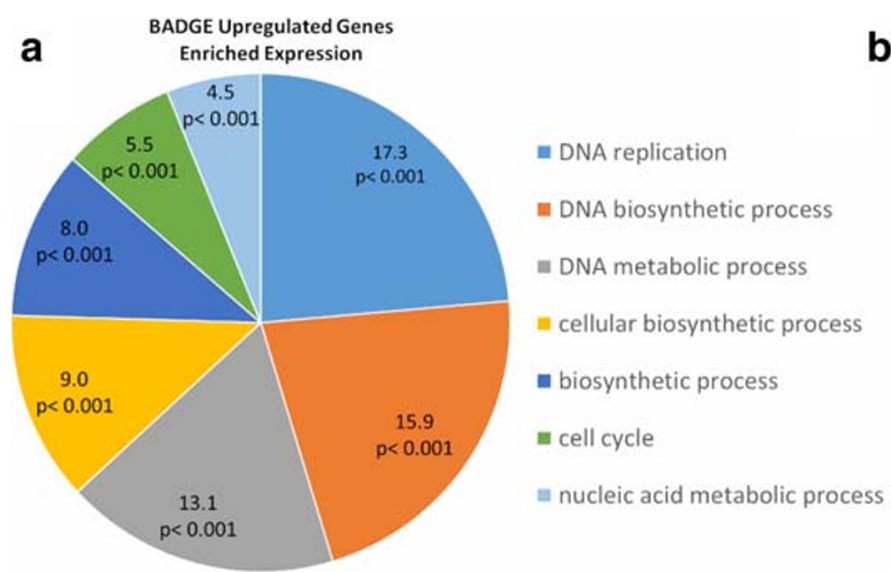

b

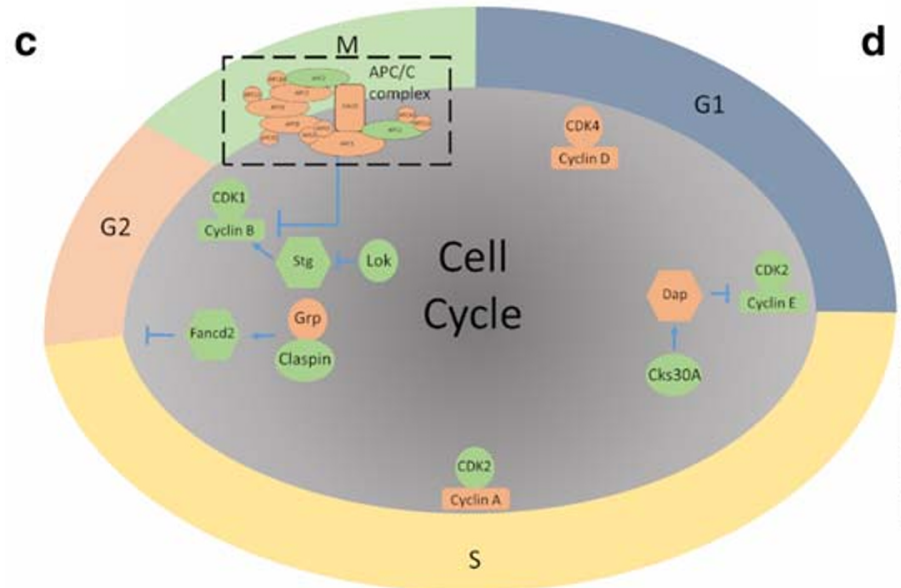

d

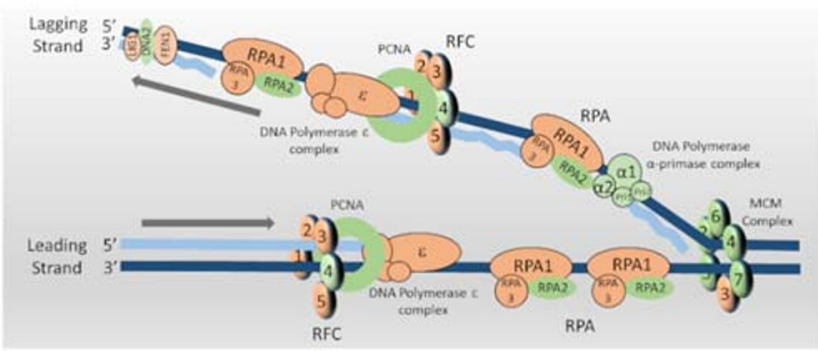

Fig. 1 BADGE increases transcript numbers of genes linked to cell proliferation. a Pie chart showing PANTHER classification of genes whose transcript number significantly increased in male Drosophila upon chronic BADGE exposure throughout development (number indicates the percentage of total BADGE up- or downregulated genes, $p$ value indicates this is significant when compared with the total number of genes in the genome linked to this category). b Many of the genes with

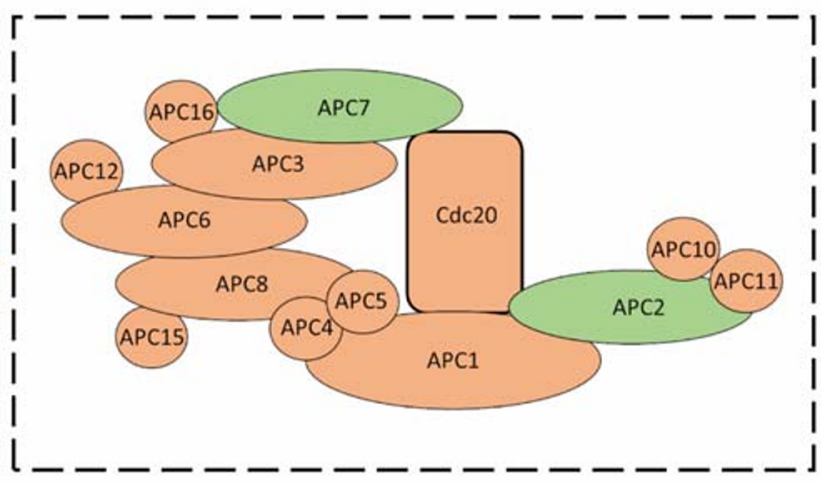

males in our experiments. By mapping the Drosophila transcriptome to the reference genome obtained from FlyBase (build dmel_r5.47_FB2012_05), 15,147 transcripts were identified, including expressed genes (mRNA), miRNA, snRNA, snoRNA, and tRNA. After performing pair-wise comparisons between all sequenced replicates and taking into consideration the "false discovery rate" and correcting for it using the Benjamini-Hochberg correction, 338 genes were differentially expressed (Supplementary Dataset S1).

Using PANTHER and DAVID, we categorized the differentially expressed genes by function (Huang da et al. 2009a, b; Mi et al. 2013). Interestingly, flies raised on food containing BADGE at the NOAEL level showed a notable enrichment in genes connected with DNA proliferation and cell cycle regulation (Fig. 1a-d and Table 1). Among the DNA proliferation genes having a significant increase in transcript copy number, Pol $\alpha$-primase and mini-chromosome maintenance $(\mathrm{Mcm})$ genes were highly enriched (Fig. $1 \mathrm{~b}$ and Table 1). Many cell

increased transcript numbers were linked to DNA replication. c Another group of genes whose transcript number was significantly increased in flies raised on BADGE-containing food was those regulating cell cycle. $\mathbf{d}$ Blow-up of the APC complex outlined with a dashed box in (c). b-d Proteins in green bubbles were transcriptionally enhanced after BADGE feeding while those in orange were not significantly affected

cycle genes having a significant increase in transcript copy number were linked to the control of $\mathrm{G}_{1}-\mathrm{S}$ and $\mathrm{G}_{2}-\mathrm{M}$ transitions (Fig. 1c, d and Table 1).

By using PANTHER and DAVID to analyze genes having a significant decrease in transcript copy number, we were able to determine the largest set belonged to the cellular response to light stimulus and the response to oxygen levels (Supplementary Figure 1). Furthermore, many genes with a significant decrease in copy number were involved in regulating metabolism or neuronal interactions (Supplementary Figure 1).

\section{BADGE feeding interferes with cell cycle genes at the transcriptional level}

To confirm the effect of BADGE on cell cycle, we selectively tested the transcriptional expression of some important cell cycle regulatory genes that had a significant increase in 
Table 1 Genes whose transcript copy number was significantly increased in Drosophila males raised on BADGE-containing food throughout the development

\begin{tabular}{|c|c|c|c|}
\hline Gene ID & Symbol & Human ortholog & Log2 (fold_change) \\
\hline \multicolumn{4}{|l|}{ DNA proliferation } \\
\hline FBgn0020633 & $\mathrm{Mcm} 7$ & MCM7 & 3.24 \\
\hline FBgn0017577 & Mcm5 & MCM5 & 3.06 \\
\hline FBgn0014861 & $\mathrm{Mcm} 2$ & MCM2 & 2.74 \\
\hline FBgn0040290 & RecQ4 & RECQL4 & 2.52 \\
\hline FBgn0005655 & PCNA & PCNA & 2.32 \\
\hline FBgn 0031540 & Pif1 & PIF1 & 2.26 \\
\hline FBgn0030170 & CG2990 & DNA2 & 2.05 \\
\hline FBgn0259113 & DNApol-alpha180 & POLA1 & 2.02 \\
\hline FBgn0025815 & Mcm6 & MCM6 & 1.85 \\
\hline FBgn0005696 & DNApol-alpha73 & POLA2 & 1.83 \\
\hline FBgn0259676 & DNApol-alpha60 & PRIM2 & 1.81 \\
\hline FBgn0086695 & hd & DONSON & 1.63 \\
\hline FBgn0043002 & Chrac-14 & POLE3 & 1.43 \\
\hline FBgn0015929 & dpa & MCM4 & 1.41 \\
\hline FBgn0260985 & $\mathrm{RfC} 4$ & RFC4 & 1.4 \\
\hline FBgn0032906 & RPA2 & RPA2 & 1.31 \\
\hline FBgn0027903 & Pol31 & POLD2 & 1.15 \\
\hline \multicolumn{4}{|c|}{ Cell cycle regulation } \\
\hline FBgn0001120 & gnu & & 7.23 \\
\hline FBgn0010097 & gammaTub37C & TUBB3 & 5.73 \\
\hline FBgn0000927 & $\mathrm{fs}(1) \mathrm{Ya}$ & & 4.77 \\
\hline FBgn0003525 & stg & $\mathrm{CDC} 25 \mathrm{C}$ & 4.19 \\
\hline FBgn0052251 & Claspin & CLSPN & 3.94 \\
\hline FBgn0000826 & png & NEK8 & 3.76 \\
\hline FBgn0029879 & APC7 & ANAPC7 & 2.83 \\
\hline FBgn0019686 & lok & CHEK2 & 2.41 \\
\hline FBgn0004107 & $\mathrm{Cdk} 2$ & CDK2 & 2.13 \\
\hline FBgn0013548 & 1(2)dtl & DTL & 2.03 \\
\hline FBgn0000405 & $\mathrm{CycB}$ & CCNB1 & 1.81 \\
\hline FBgn0003114 & plu & POTEA & 1.69 \\
\hline FBgn0034403 & CG18190 & MAPRE2 & 1.56 \\
\hline FBgn0010382 & $\mathrm{CycE}$ & CCNE1 & 1.52 \\
\hline FBgn0051658 & Nnflb & & 1.35 \\
\hline FBgn0032105 & borr & CDCA8 & 1.24 \\
\hline FBgn0038827 & Fancd2 & FANCD2 & 1.20 \\
\hline FBgn0004106 & Cdk1 & CDK1 & 1.17 \\
\hline FBgn0039638 & dgt6 & HAUS6 & 1.17 \\
\hline FBgn0264296 & CG43774 & ANAPC2 & 1.14 \\
\hline FBgn0263979 & Caf1-55 & RBBP4 & 1.05 \\
\hline FBgn0030500 & Ndc80 & NDC80 & 1.04 \\
\hline FBgn0010314 & Cks30A & CKS2 & 1.02 \\
\hline
\end{tabular}

transcript copy number in our sequencing experiment. Flies were maintained throughout the development on three BADGE dosages, $20 \mu \mathrm{M}, 60 \mu \mathrm{M}$, and $200 \mu \mathrm{M}$, as well as control food. As before, $\mathrm{F}_{1}$ males were collected and aged 5-7 days before sampling. Consistent with the transcriptome sequencing data, transcripts of Cyclin $B(C y c B)$, pan gu (png), and string (stg) were significantly increased in flies maintained on food containing $200 \mu \mathrm{M}$ BADGE (approximately 50\%) (Fig. 2a, c, d). Cyclin $E(C y c E)$ transcript was significantly increased in flies raised on both $60 \mu \mathrm{M}$ and 

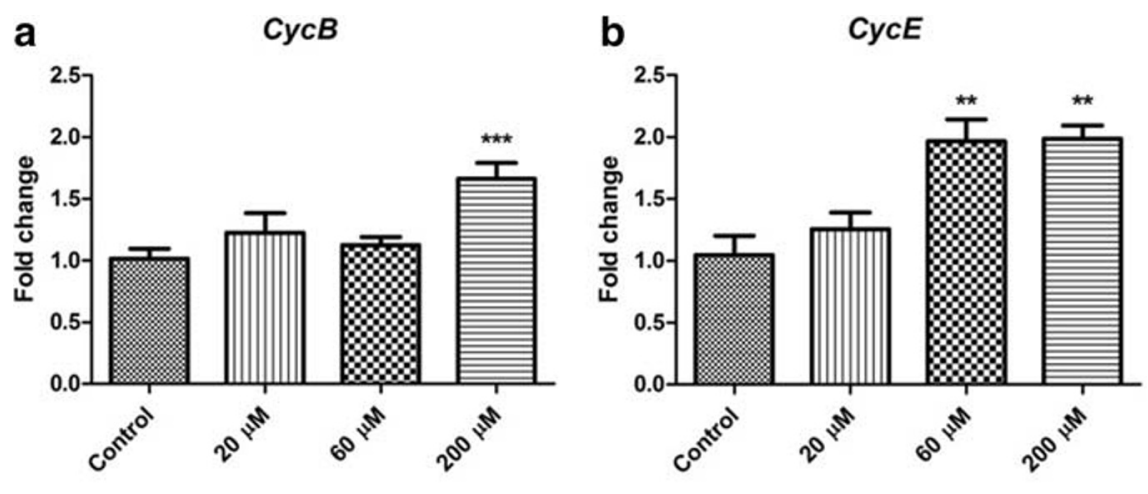

C

stg
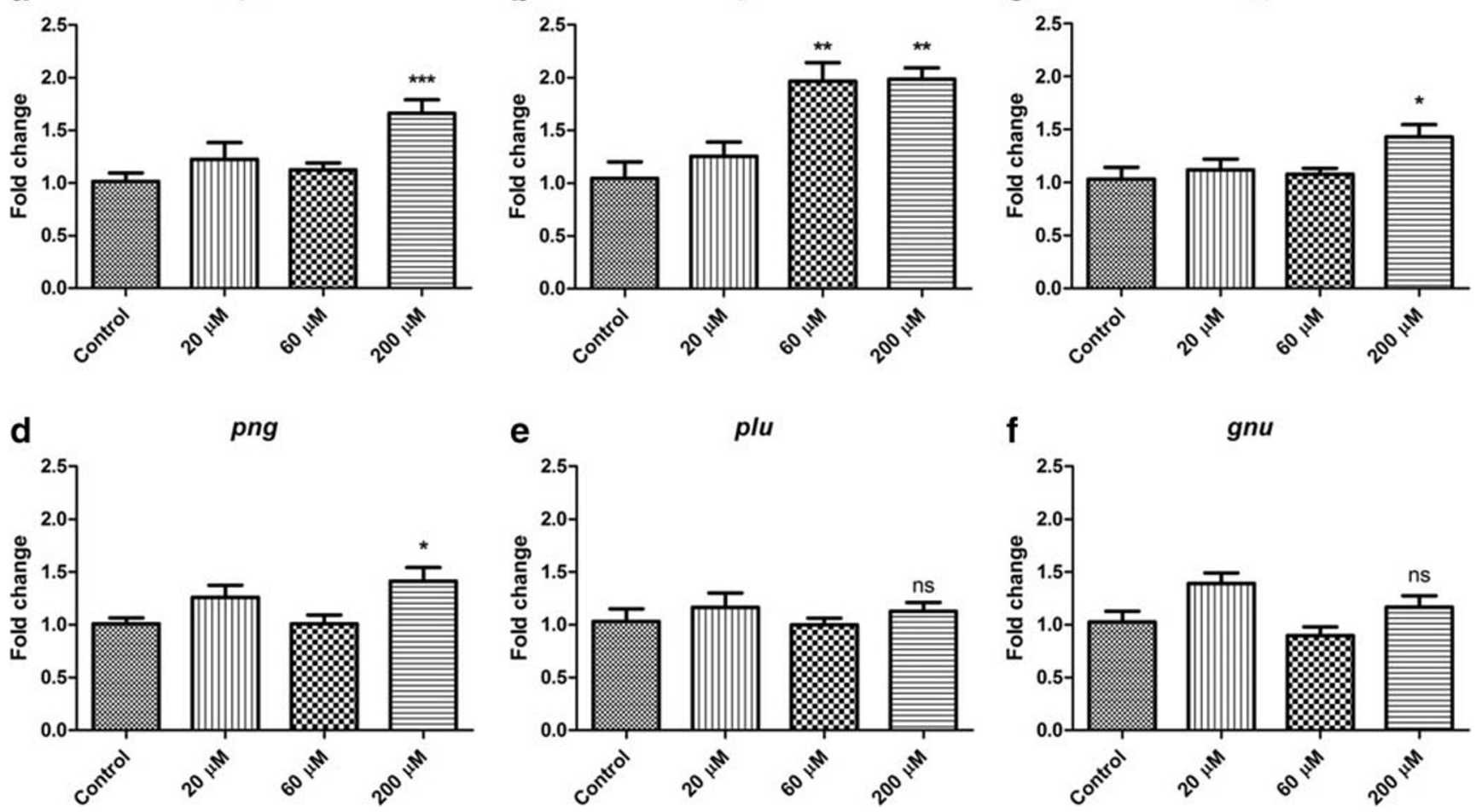

Fig. 2 BADGE feeding causes an increase in cell cycle gene expression. CSORC flies were raised on either ethanol-control food or BADGE food and aged for 5-7 days before sampling. The flies were kept at $-80{ }^{\circ} \mathrm{C}$ for long-term storage if necessary. RNA was carefully extracted from whole flies and converted to cDNA before performing qRT-PCR ( $n=6$ biological replicates for each treatment and 10 male flies were used for each biological sample). Data showing that $C y c B(\mathbf{a}), \operatorname{stg}(\mathbf{c})$, and $p n g$

(d) were notably increased in $200 \mu \mathrm{M}$ BADGE-fed flies and $C y c E$ (b) was increased in both $60 \mu \mathrm{M}$ and $200 \mu \mathrm{M}$ BADGE-fed flies. However, the expressions of $p l u(\mathbf{e})$ and $g n u(\mathbf{f})$ were not significantly influenced by BADGE $(* p<0.05, * * * p<0.005$ compared with ethanol-control feeding, one-way ANOVA with Tukey's post hoc test for multiple comparisons was applied. Error bar represented SEM)

$200 \mu \mathrm{M}$ BADGE (Fig. 2b). However, the transcript levels of two components of the png-kinase complex, gluon $(g l u)$ and giant nuclei (gnu), were not changed (Fig. 2e, f). The inconsistency can result from the different sensitivities and procedures between whole transcriptome sequencing and quantitative RT-PCR (qPCR). Together, the two methodologies, sequencing and $\mathrm{qPCR}$, verified that chronic exposure to BADGE during development upregulates cell cycle genes in Drosophila.

\section{BADGE exposure leads to over-proliferation of larval hemocytes}

The Drosophila larval hematopoietic system is suitable for assessing cell proliferation (Zettervall et al. 2004). Therefore, we employed this system to test the effect of BADGE on cell proliferation. As with the whole transcriptome sequencing, we used our laboratory wild-type CSORC strain and maintained the larvae on BADGEcontaining food. All food, including for controls, was dyed with red food coloring. In this way, we were able to distinguish early and late wandering third instar larvae, which is important when comparing circulating hemocyte numbers
(Zettervall et al. 2004). The number of circulating hemocytes increases rapidly during development; therefore, by using the red food coloring, we were able to stage wandering larvae according to the presence (early wandering third instar larvae) or absence (late wandering third instar larvae) of food in the gut (Zettervall et al. 2004). Late wandering third instar larvae were bled on a glass slide and stained with DAPI to visualize the nuclei. ImageJ was then employed to count the number of hemocytes (Howell et al. 2012). Compared with control flies, larvae raised on $20 \mu \mathrm{M}$ and $60 \mu \mathrm{M}$ BADGE did not induce a significant increase in the number of circulating hemocytes. However, raising larvae on food containing $200 \mu \mathrm{M}$ BADGE caused a $42 \%$ increase $(p<0.05)$ in the number of circulating hemocytes (Fig. 3a).

Next, we employed the GAL4-UAS system to sensitize hemocyte proliferation in larvae. The Hml-GFP driver, which expresses both GAL4 and GFP specifically in hemocytes (Goto et al. 2003), was crossed to the UAS-Ras ${ }^{V 12}$ strain, to overexpress constitutively active Ras GTPase. This should cause the Hml-GFP>UAS-Ras ${ }^{V 12}$ larvae to be sensitive to agents that influence proliferation (Asha et al. 2003). In line with our assumption, unlike wild-type larvae, where hemocyte numbers only significantly increased when flies were raised 


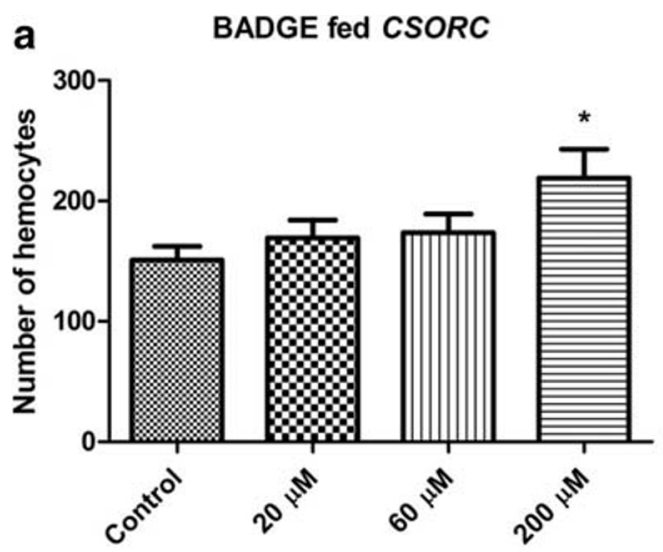

Fig. 3 BADGE causes over-proliferation of larval hemocytes. We employed the hematopoietic system to examine the effect of BADGE inducing cell over-proliferation in vivo. Third instar larvae were selected to perform the bleeding assay. a CSORC larvae were bled to test if BADGE can cause significant cell proliferation. At least 15 larvae were bled and the number of hemocytes was counted for each concentration. Compared with the controls, larvae fed on $200 \mu \mathrm{M}$ BADGE had significantly more hemocytes $(\sim 40 \%)$ while $20 \mu \mathrm{M}$ and $60 \mu \mathrm{M}$ were not influenced. b To confirm our result, we employed the GAL4-UAS system by crossing Hml-GFP with UAS-Ras ${ }^{V 12}$ to

on food containing $200 \mu \mathrm{M}$ BADGE, the Hml-GFP>UAS$\operatorname{Ras}^{v 12}$ larvae exhibited significant cell over-proliferation at lower concentrations $(20 \mu \mathrm{M}$ and $60 \mu \mathrm{M})$, when compared with $H m l-G F P>U A S-$ Ras $^{v 12}$ larvae raised on normal food (Fig. 3b). In fact, the normalized hemocyte fold-changes in circulating cell numbers were elevated approximately $60 \%$ $(p<0.005)$ and $100 \%(p<0.005)$, respectively (Fig. 3b). Again, this increase is compared with Hml-GFP $>$ UAS-Ras ${ }^{12}$ larvae raised on normal food, which have significantly higher numbers of circulating hemocytes than wild type larvae (Asha et al. 2003).

\section{BADGE does not damage hematopoietic tissue}

We performed live imaging with $H m l-G F P$ larvae to exclude the possibility that BADGE induces the release of a pool of sessile hemocytes attached to the epidermis in each larval segment. Since there is a GFP signal in the hemocytes, these cells are easily visible under fluorescent microscopy. For this experiment, we exchanged the NOAEL mimicking $20 \mu \mathrm{M}$ concentration for larvae raised on food containing $135 \mu \mathrm{M}$ BADGE, in order to understand if BADGE was capable of disrupting the hematopoietic tissue. Consequently, Hml-GFP larvae were raised on food containing $0 \mu \mathrm{M}$ (Fig. 4a), $60 \mu \mathrm{M}$ (Fig. 4b), $135 \mu \mathrm{M}$ (Fig. 4c), or $200 \mu \mathrm{M}$ (Fig. 4d) of BADGE. At all BADGE concentrations, the morphological structure of the segmentally defined epidermis-attached sessile hemocytes was intact (Fig. 4b-d).

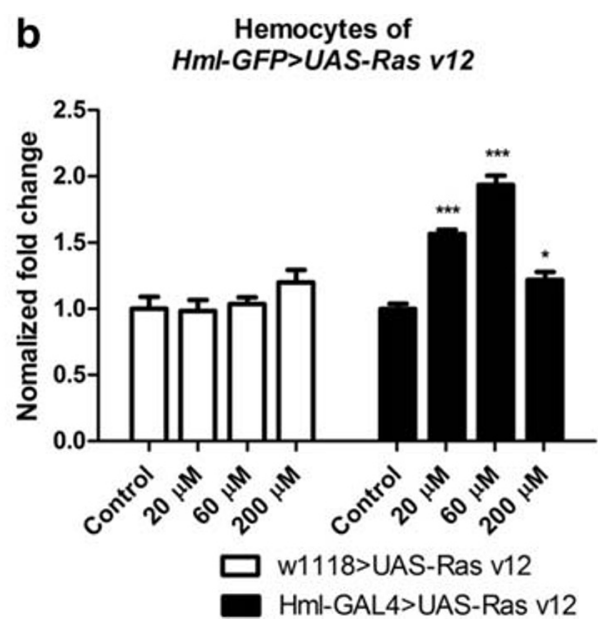

overexpress Ras in blood cells. Additionally, all data were normalized to corresponding control fed flies, which could eliminate the difference between hemocyte amounts between the two groups. After BADGE diet and bleeding, the Hml-GFP>UAS-Ras V12 flies, compared with $w^{1118}>$ $U A S-$ Ras $^{V 12}$ controls, showed an obvious increase in the number of circulating hemocytes on $20 \mu \mathrm{M}, 60 \mu \mathrm{M}$, and $200 \mu \mathrm{M}$ BADGE food $(* p<0.05, * * * p<0.005$ compared with ethanol-controls, one-way ANOVA with Tukey's post hoc test for multiple comparisons was applied. Error bars represent SEM)

\section{Discussion}

In Drosophila males, chronic exposure to BADGE throughout developmental causes a significant change in the transcript number of 338 genes. The expressions of genes connected to DNA polymerization and cell cycle control were particularly enriched among these transcripts. Many genes whose transcript number significantly decreased were connected to neuronal signaling and synapsis homeostasis. For a subset of genes connected to cell cycle control, we confirmed the increased transcript number by using quantitative real-time PCR. In relation to the significant number of cell proliferation genes with increased transcript number, we also demonstrate that late third instar larvae raised on BADGE-containing food have a significantly increased number of circulating hemocytes, without any obvious physical lesion of a hematopoietic tissue, known as the lymph gland in Drosophila, or a pool of sessile hemocytes attached to the larval epidermis. Furthermore, the susceptibility of the hematopoietic system to BADGE exposure can be sensitized in a background where dominant active Ras GTPase is expressed specifically in hemocytes.

In the current study, we applied both genetic and phenotypic studies to reveal the cell proliferative effect of chronic BADGE exposure at a level comparative to the human NOAEL. Of note, in a breast cancer cell line (T47D), it was observed that BADGE induced cellular proliferation (Nakazawa et al. 2002). Other studies have also hinted at the proliferative effects of BADGE exposure in cell lines (Suarez et al. 2000; Sueiro et al. 2006). However, in all of those 
Fig. 4 BADGE does not disturb the sessile hemocyte population. The increase in circulating hemocytes can be caused by the release of a sessile population attached to the epidermis; therefore, we used confocal microscopy to evaluate the effect of BADGE on this hematopoietic tissue. The Hml-GFP flies were raised and allowed to lay eggs on either BADGE or control food.

Living $F_{1}$ larvae were anesthetized on ice and fix by nail polish on a glass slide. Images were taken immediately. Data show larvae raise on food containing $0 \mu \mathrm{M}$ (a), $60 \mu \mathrm{M}$ (b), $135 \mu \mathrm{M}(\mathbf{c})$, and $200 \mu \mathrm{M}$ (d) BADGE. No morphological changes were observed in the sessile population; the sessile bands were clear and intact in each larval segment
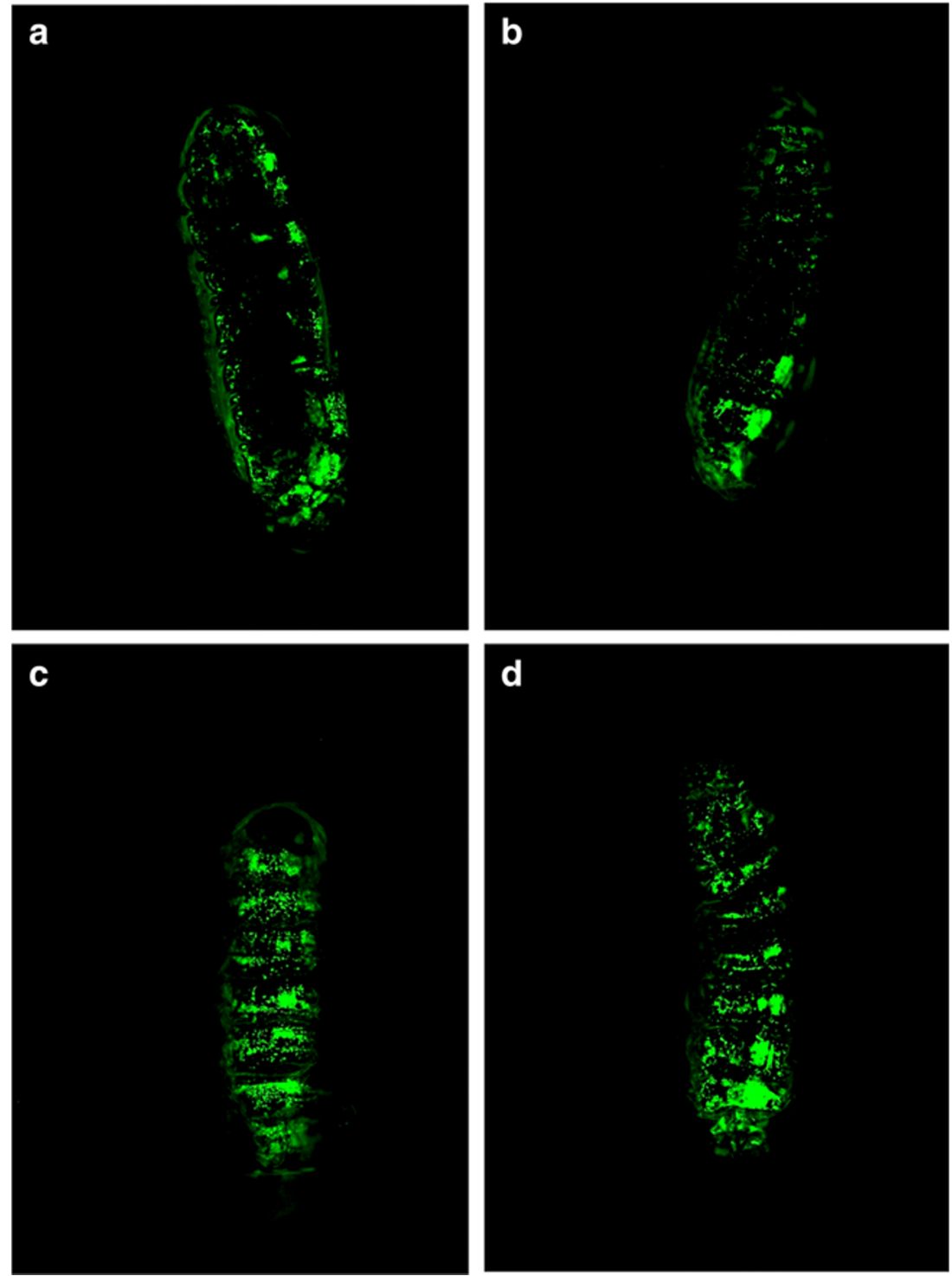

studies, in vivo data was missing. Interestingly, our whole transcriptome sequencing data, using adult male Drosophila, demonstrates that exposure to BADGE throughout development causes a significant increase in the transcript number of genes linked to DNA replication and cell cycle control (see Fig. 1). For example, the expressions of $C y c B, C y c E$, and $s t g$ genes are significantly increased, which gives hints that cell replication may be abnormal.

We provide in vivo phenotypic evidence demonstrating the ability of BADGE to induce cell proliferation. In recent years, the Drosophila hematopoietic system has been increasingly used in inflammatory and cell proliferation-related studies (Milton et al. 2014; Wang et al. 2014; Zettervall et al. 2004). With the advantages of the hematopoietic system, we report a notable increase in the number of circulating hemocytes in late third instar larvae maintained on BADGE-containing food throughout development, which is consistent with the RNA- seq and qPCR results. This not only demonstrates that genes involved in regulating cell proliferation are transcriptionally more active, but also that cell proliferation in at least one system (hematopoietic) is significantly increased upon chronic exposure to BADGE. The peroxisome proliferator-activated receptor-gamma (PPAR $\gamma$ ) has been linked to BADGE responses (Chen et al. 2019; Dworzanski et al. 2010; Nakamuta et al. 2002). However, there is no obvious PPAR $\gamma$ homolog in Drosophila, which suggests the involvement of additional molecular pathways that need to be identified in the future.

Although BADGE is detected at a low level in some epoxy resin-based products, the influence of chronic and developmental exposure needs to be considered. Some surveys reported that daily BADGE intake in humans is much lower than the NOAEL level of $15 \mathrm{mg} / \mathrm{kg}$ body weight/day (reviewed in (Poole et al. 2004)). However, we believe these results do not go against our study, as humans are exposed to multiple 
xenobiotic chemicals via various routes over an extended period of time. The continual interaction between different substrates may have an influence on the final exposure outcomes.

\section{Conclusions}

We report that chronic BADGE exposure throughout development at a level equivalent to the human NOAEL increases the transcript copy number of genes related to cell proliferation and causes hemocyte proliferation in Drosophila larvae. Furthermore, we show that feeding BADGE to larvae whose cells are already sensitized for cell division by the expression of a dominant active Ras (Asha et al. 2003) significantly increased the number of circulating hemocytes at a BADGE concentration 100-fold lower than non-sensitized cells. Due to the ubiquitous exposure to such xenobiotics in modern society, it is important to elucidate a molecular mechanism for the effect of BADGE on cell proliferation in vivo.

Acknowledgements Open access funding provided by Uppsala University.

Author contributions Conceived and designed the experiments: MJW, HC, and HBS. Performed the experiments: HC, TL, and TJM. Analyzed the data: HC, TL, TJM, MJW, and HBS. Contributed reagents/materials/analysis tools: MJW and HBS. Wrote the paper: MJW, $\mathrm{HC}$, and HBS.

Funding information This study was supported by the Swedish Research Council, Formas, and the Novo Nordisk Foundation.

\section{Compliance with ethical standards}

Competing interests The authors declare that they have no conflict of interests.

Open Access This article is licensed under a Creative Commons Attribution 4.0 International License, which permits use, sharing, adaptation, distribution and reproduction in any medium or format, as long as you give appropriate credit to the original author(s) and the source, provide a link to the Creative Commons licence, and indicate if changes were made. The images or other third party material in this article are included in the article's Creative Commons licence, unless indicated otherwise in a credit line to the material. If material is not included in the article's Creative Commons licence and your intended use is not permitted by statutory regulation or exceeds the permitted use, you will need to obtain permission directly from the copyright holder. To view a copy of this licence, visit http://creativecommons.org/licenses/by/4.0/.

\section{References}

Asha H, Nagy I, Kovacs G, Stetson D, Ando I, Dearolf CR (2003) Analysis of Ras-induced overproliferation in Drosophila hemocytes. Genetics 163:203-215
Chen J et al (2019) Netrin-1 alleviates subarachnoid haemorrhageinduced brain injury via the PPARgamma/NF-KB signalling pathway. J Cell Mol Med 23:2256-2262. https://doi.org/10.1111/jcmm. 14105

Climie IJ, Hutson DH, Stoydin G (1981) Metabolism of the epoxy resin component 2,2-bis[4-(2,3-epoxypropoxy)phenyl] propane, the diglycidyl ether of bisphenol a (DGEBPA) in the mouse. Part II. Identification of metabolites in urine and faeces following a single oral dose of 14C-DGEBPA Xenobiotica; the fate of foreign compounds in biological systems. 11:401-424. https://doi.org/10.3109/ 00498258109045851

Dworzanski T, Celinski K, Korolczuk A, Slomka M, Radej S, Czechowska G, Madro A, Cichoz-Lach H (2010) Influence of the peroxisome proliferator-activated receptor gamma (PPAR-gamma) agonist, rosiglitazone and antagonist, biphenol-A-diglicydyl ether (BADGE) on the course of inflammation in the experimental model of colitis in rats. J Physiol Pharmacol 61:683-693

Geyer R, Jambeck JR, Law KL (2017) Production, use, and fate of all plastics ever made. Sci Adv 3:e1700782. https://doi.org/10.1126/ sciadv. 1700782

Goto A, Kadowaki T, Kitagawa Y (2003) Drosophila hemolectin gene is expressed in embryonic and larval hemocytes and its knock down causes bleeding defects. Dev Biol 264:582-591

Hammarling L, Gustavsson H, Svensson K, Oskarsson A (2010) Migration of bisphenol-A diglycidyl ether (BADGE) and its reaction products in canned foods. Food Addit Contam 17(11):937-943

Howell L, Sampson CJ, Xavier MJ, Bolukbasi E, Heck MM, Williams MJ (2012) A directed miniscreen for genes involved in the Drosophila anti-parasitoid immune response. Immunogenetics 64: 155-161. https://doi.org/10.1007/s00251-011-0571-3

Huang da W, Sherman BT, Lempicki RA (2009a) Bioinformatics enrichment tools: paths toward the comprehensive functional analysis of large gene lists. Nucleic Acids Res 37:1-13. https://doi.org/10.1093/ nar/gkn923

Huang da W, Sherman BT, Lempicki RA (2009b) Systematic and integrative analysis of large gene lists using DAVID bioinformatics resources. Nat Protoc 4:44-57. https://doi.org/10.1038/nprot.2008. 211

Hyoung UJ, Yang YJ, Kwon SK, Yoo JH, Myoung SC, Kim SC, Hong YP (2007) Developmental toxicity by exposure to bisphenol A diglycidyl ether during gestation and lactation period in SpragueDawley male rats. J Prevent Med Public Health 40:155-161. https:// doi.org/10.3961/jpmph.2007.40.2.155

Jadhav RR, Santucci-Pereira J, Wang Y, Liu J, Nguyen T, Wang J, Jenkins S, Russo J, Huang T, Jin V, Lamartiniere C (2017) DNA methylation targets influenced by bisphenol A and/or genistein are associated with survival outcomes in breast cancer patients. Genes:8. https:// doi.org/10.3390/genes 8050144

Jambeck JR, Geyer R, Wilcox C, Siegler TR, Perryman M, Andrady A, Narayan R, Law KL (2015) Marine pollution. Plastic waste inputs from land into the ocean. Science 347:768-771. https://doi.org/10. 1126/science. 1260352

Mi H, Muruganujan A, Casagrande JT, Thomas PD (2013) Large-scale gene function analysis with the PANTHER classification system. Nat Protoc 8:1551-1566. https://doi.org/10.1038/nprot.2013.092

Milton CC, Grusche FA, Degoutin JL, Yu E, Dai Q, Lai EC, Harvey KF (2014) The Hippo pathway regulates hematopoiesis in Drosophila melanogaster. Curr Biol 24:2673-2680. https://doi.org/10.1016/j. cub.2014.10.031

Nakamuta $\mathrm{M}$ et al (2002) Bisphenol a diglycidyl ether (BADGE) suppresses tumor necrosis factor-alpha production as a PPARgamma agonist in the murine macrophage-like cell line, RAW 264.7. Cell Biol Int 26:235-241. https://doi.org/10.1006/cbir.2001.0838

Nakazawa H, Yamaguchi A, Inoue K, Yamazaki T, Kato K, Yoshimura Y, Makino T (2002) In vitro assay of hydrolysis and chlorohydroxy derivatives of bisphenol A diglycidyl ether for estrogenic activity. 
Food Chem Toxicol 40:1827-1832. https://doi.org/10.1016/s02786915(02)00165-5

Pang Q, Li Y, Meng L, Li G, Luo Z, Fan R (2019) Neurotoxicity of BPA, BPS, and BPB for the hippocampal cell line (HT-22): an implication for the replacement of BPA in plastics. Chemosphere 226:545-552. https://doi.org/10.1016/j.chemosphere.2019.03.177

Poole A, van Herwijnen P, Weideli H, Thomas MC, Ransbotyn G, Vance C (2004) Review of the toxicology, human exposure and safety assessment for bisphenol A diglycidylether (BADGE). Food Addit Contam 21:905-919. https://doi.org/10.1080/02652030400007294

Ramakers C, Ruijter J, Deprez R, Moorman A (2003) Assumption-free analysis of quantitative real-time polymerase chain reaction (PCR) data. Neurosci Lett 339:62-66. https://doi.org/10.1016/s03043940(02)01423-4

Ramilo G, Valverde I, Lago J, Vieites JM, Cabado AG (2006) Cytotoxic effects of BADGE (bisphenol A diglycidyl ether) and BFDGE (bisphenol $\mathrm{F}$ diglycidyl ether) on Caco-2 cells in vitro. Arch Toxicol 80(11):748-755

Suarez S, Sueiro RA, Garrido J (2000) Genotoxicity of the coating lacquer on food cans, bisphenol A diglycidyl ether (BADGE), its hydrolysis products and a chlorohydrin of BADGE. Mutat Res 470: 221-228. https://doi.org/10.1016/s1383-5718(00)00109-1

Sueiro RA, Suarez S, Araujo M, Garrido MJ (2006) Study on mutagenic effects of bisphenol A diglycidyl ether (BADGE) and its derivatives in the Escherichia coli tryptophan reverse mutation assay. Mutat Res 609:11-16. https://doi.org/10.1016/j.mrgentox.2006.05.012

Vandesompele J, De Preter K, Pattyn F, Poppe B, Van Roy N, De Paepe A, Speleman F (2002) Accurate normalization of real-time quantitative RT-PCR data by geometric averaging of multiple internal control genes. Genome Biol:3. https://doi.org/10.1186/gb-2002-37-research0034

Wang L, Wu Y, Zhang W, Kannan K (2012) Widespread occurrence and distribution of bisphenol A diglycidyl ether (BADGE) and its derivatives in human urine from the United States and China. Environ Sci Technol 46:12968-12976. https://doi.org/10.1021/ es304050f

Wang L, Kounatidis I, Ligoxygakis P (2014) Drosophila as a model to study the role of blood cells in inflammation, innate immunity and cancer. Front Cell Infect Microbiol 3:113. https://doi.org/10.3389/ fcimb.2013.00113

Wang L, Xue J, Kannan K (2015) Widespread occurrence and accumulation of bisphenol A diglycidyl ether (BADGE), bisphenol $\mathrm{F}$ diglycidyl ether (BFDGE) and their derivatives in human blood and adipose fat. Environ Sci Technol 49:3150-3157. https://doi. org/10.1021/acs.est.5b00096

Williams MJ, Goergen P, Rajendran J, Klockars A, Kasagiannis A, Fredriksson R, Schioth HB (2013) Regulation of aggression by obesity-linked genes TfAP-2 and Twz through octopamine signaling in Drosophila. Genetics. https://doi.org/10.1534/genetics.113. 158402

Williams MJ et al (2016) The Drosophila ETV5 homologue Ets96B: molecular link between obesity and bipolar disorder. PLoS Genet 12:e1006104. https://doi.org/10.1371/journal.pgen.1006104

Yonekubo J, Hayakawa K, Sajiki J (2008) Concentrations of bisphenol a, bisphenol a diglycidyl ether, and their derivatives in canned foods in Japanese markets. J Agric Food Chem 56:2041-2047. https://doi. org/10.1021/jf073106n

Zettervall C-J, Anderl I, Williams MJ, Palmer R, Kurucz E, Ando I, Hultmark D (2004) A directed screen for genes involved in Drosophila blood cell activation. PNAS 101:14192-14197

Zhu R-J, Wu M-Q, Li Z-J, Zhang Y, Liu K-Y (2013) Hematopoietic recovery following chemotherapy is improved by BADGEinduced inhibition of adipogenesis. Int J Hematol 97(1):58-72

Publisher's note Springer Nature remains neutral with regard to jurisdictional claims in published maps and institutional affiliations. 